\title{
AC 2012-2974: ENGINEERING LABORATORY ENHANCEMENT THROUGH CLOUD COMPUTING
}

\section{Dr. Lin Li, Prairie View A\&M University}

Lin Li is an Assistant Professor of the Computer Science Department at Prairie View A\&M University. He received his Ph.D. in computer science from the University of Nebraska, Lincoln in 2004. Before that, he received his B.S. and M.E. from Beijing Institute of Technology and Chinese Academy of Sciences in 1996 and 1999, respectively. Currently, his research interests are in computer educational technology, green home, and network communications.

\section{Prof. Yongpeng Zhang, Prairie View A\&M University}

Yongpeng Zhang received his Ph.D. degree in electrical engineering from University of Houston (2003), and then joined DSP Solutions Lab of CECSTR, Prairie View A\&M University as a post-doctoral research fellow. Currently, he is a tenured Associate Professor in the Engineering Technology Department, Prairie View A\&M University, Texas. His research interests are control system, power electronics, motor drive, mechatronics, etc. His research has been widely recognized and supported by multiple grants from Army, NSF, and industry.

\section{Dr. Lei Huang, Prairie View A\&M University}

Lei Huang is an Assistant Professor in the Computer Science Department, Prairie View A\&M University. He received his Ph.D. in Computer Science from University of Houston in 2006. His research areas are in high performance computing, cloud computing, programming model, and compiler optimizations. 


\title{
Engineering Laboratory Enhancement through Cloud Computing
}

\begin{abstract}
Laboratories are important to engineering and technology curricula. Through systematically designed experiments, students can gain hands-on experience, enhance classroom learning, and cultivate career interests. However, traditional laboratory conduction is often restricted by various reasons such as facility cost, conflicted schedule, and limited space. Thus, how to effectively extend laboratories via cyberspace and maximize resources utilization has caused many researchers' attention.
\end{abstract}

In recent years, Cloud Computing technology has developed drastically, which provided an ideal solution for virtual and remote laboratory implementation. This paper presents a project currently conducted at Prairie View A\&M University. Using Virtual Computing Lab (VCL), a Cloud Computing application developed by North Carolina State University and IBM, the investigators made a series of LabVIEW based engineering laboratories online. These laboratory setups greatly reduce the cost of experimental facilities, improve the lab schedule function, increase the accessibility of equipment and courseware, and support teachers' instructional needs. At the same time, the original nature and underlying contents of the laboratories are stilled retained. This paper elaborates the infrastructure of the renovated laboratories as well as the current progress and implementation strategies of the project.

\section{Background}

Laboratories and experiments play a vital role in Engineering Technology (ET) education. ET program features hands-on training through which students can investigate the nature of science and engineering, observe dynamic phenomena, test hypotheses, and develop problem-solving and implementation skills. Traditionally, engineering laboratories are conducted in lab classrooms or workshops. However, restrictions arising from equipment cost, room arrangement, and scheduling often hinder the smooth conduction of laboratories. Therefore, how to effectively carry out lab activities and maximize the usage of limited resources becomes the key to address these concerns.

The fast development of Information Technology drastically impacted engineering education and facilitated the invention of various courseware and learning tools. It also enabled online course conveyance. Studies ${ }^{1}$ indicated that "over 5.6 million students were taking at least one online course during the fall 2009 term, an increase of nearly one million over the number reported the previous year". Correspondingly, how to extend the traditional engineering laboratories over the Internet caused many researchers' attention ${ }^{2,3,4}$. In general, online laboratories can be categorized into two types: virtual environment emulation and remote instrument control through internet access. Compared with the traditional counterparts, virtual and remote labs (VR-Labs) have intrinsic merits in cost and convenience.

In recent years, Cloud Computing has attracted much attention from industry and academia ${ }^{5,6}$. As a sophisticated infrastructure, Cloud Computing provides on-demand computing for anyone within a network connection using a third party service (web service). Its advantage lies in the 
accessibility of the data and applications from anywhere, at anytime, and with any web-allowed device. This feature of Cloud eliminates the costs and complexity associated with acquiring and managing the information infrastructure. Cloud Computing also provides scalable and elastic computing resources that are served from the shared resource pool which can be either physical or virtual. Due to these characteristics, Cloud Computing has been developed to be a successful business model.

By leveraging an established Cloud infrastructure, the authors are exploring the renovation of engineering labs by integrating Cloud Computing to optimize lab equipment utilization and enhance student learning effectiveness.

\section{Previous Work and Existing Remote Engineering Laboratory at PVAMU}

In 2009, one of the authors and two other scholars in the University of Houston and Texas Southern University initiated the research on remote virtual lab conduction. Based on LabVIEW remote engine, their courseware uses an in-house developed scheduler web server (SWS) to bridge physical laboratory experiments with the Internet end users. The courseware is integrated with account management and scheduling functions through which users can reserve lab equipment and time slots. After that, a remote experiment doer can manipulate lab instruments and focus on class contents, with guaranteed time and resources. This system is independent of lab equipment and experimental technology.

Practical lab deployment of the courseware showed that it was efficient in improving the resource utilization ${ }^{4,7,8}$. Meanwhile, we also noticed some potential issues. As shown in Figure 1, in this remote engineering laboratory framework, a user connects to the scheduler web server and requests to use the LabVIEW application on the experiment servers. Scheduler web server takes charge of managing and scheduling the user's requests, and communicates with the experiment servers. The functionality is constrained by the capability of web browser and Java script since all work needs to be done on the web browser. All of users share the single web server, which could become a bottleneck when there are a large number of usages.

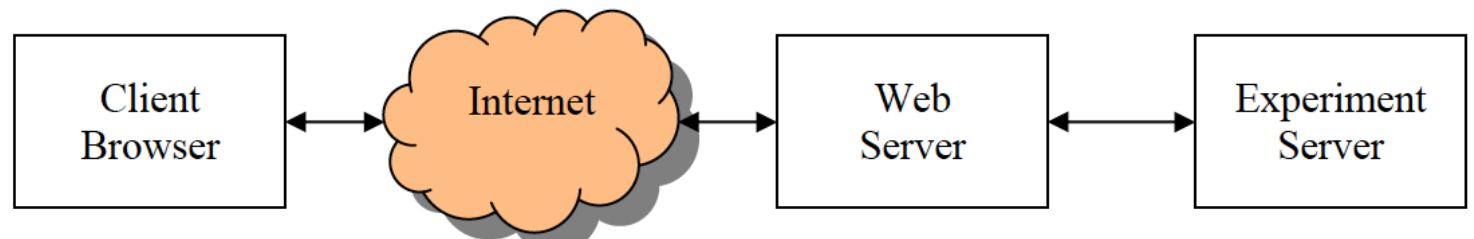

Figure 1. The existing remote engineering laboratory framework

\section{PVAMU Cloud System}

To further improve the remote lab implementation, we are working on new lab architectures to overcome the limitations. This on-project is built based on our experience of setting up a Cloud Computing environment at PVAMU for supporting students to utilize a variety of software in their classes. The Computer Science Department at PVAMU received four IBM Blade Center systems donated by IBM in 2009, and used it as the foundation to establish the Cloud Computing Simulation Laboratory that was officially open to public use in April 2010. The Cloud 
Computing system consists of 4 IBM Blade Centers. Each of them consists of 14 blade nodes, and each node is equipped with two $2.4 \mathrm{GHz}$ CPUs, one 40GB hard disk, and 2.5 or 4 GB Ram. The entire system consists of 56 low power nodes, which are hosted in one 42U IBM enterprise rack.

The department has deployed the Virtual Computing Lab developed by North Carolina State University on the IBM Blade Center. VCL is used to manage the Cloud Computing system, and delivers dedicated compute environments according to users' requests. A conceptual view of the VCL based Cloud Computing environment is depicted in Figure 2, which is referred from the VCL system guide. VCL defines and manages a pool of virtual machines created by using VMware, physical computers and resources, and a set of customized operating systems and applications (called images). Through VCL built-in resource scheduling system, a user can connect to the VCL web portal to request access to a desired application environment for a limited time. It provides convenient ways of usage and the availability of software without physically being at the lab, classroom, office, or public library ${ }^{9,10}$. Software resources are accessible to all students and faculty at PVAMU. This system is a highly reusable, sustainable, scalable, and customizable.

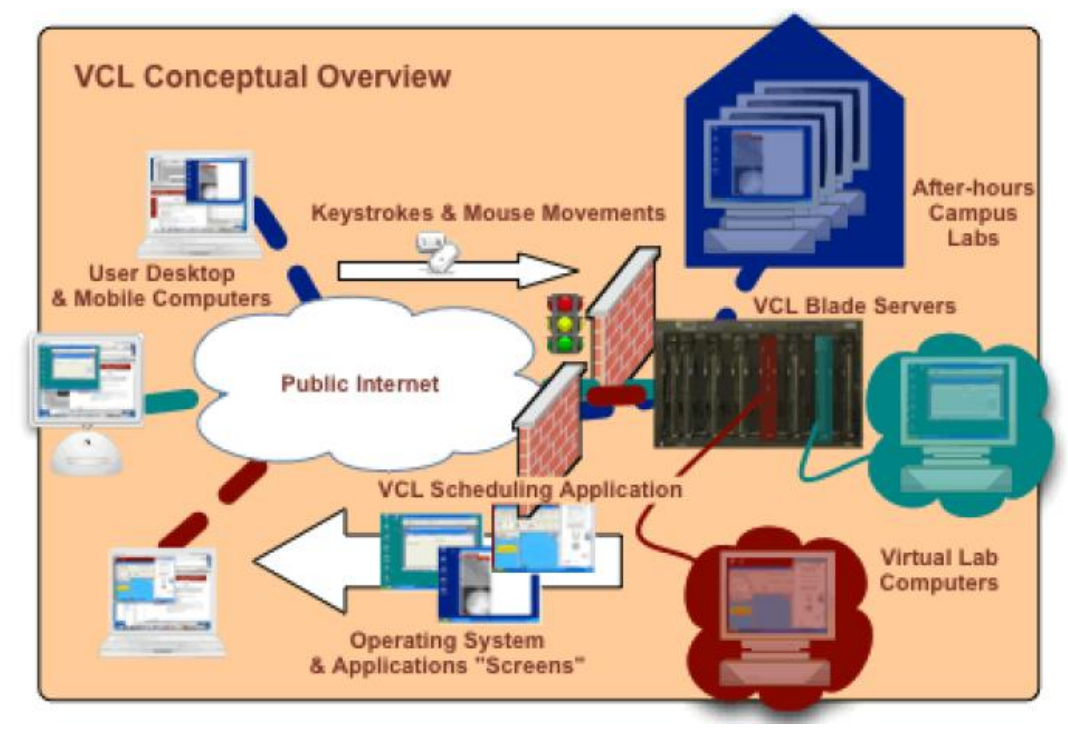

Figure 2. A conceptual view of VCL infrastructure

\section{Building Engineering Labs on VCL Cloud}

Based on previous research and instructional needs, we believe that it is beneficial to build the engineering laboratory on the PVAMU Cloud system to take advantage of the Cloud Computing technology. Figure 3 presents an overview of the new Cloud-based engineering remote laboratory. In this system, we utilize the sophisticated Cloud management and scheduling functionality to provide a secured, scalable, and dedicated remote laboratory environment to every user. To do it, we create a remote laboratory virtual machine, set the required hardware configuration and user access privilege, and store it in the image repository of the Cloud. The Cloud manages the image and finds the available and best-suited hardware environment to launch the image when a request comes in. A user needs to register in the Cloud system first before making any requests. After registration, a user is able to make a reservation to the remote 
lab image at any time, and anywhere. A user can request to use the remote lab immediately or at a specified time. The Cloud launches the image based on the available resources and notifies the user once it is ready. The user can then log into the dedicated environment and conduct experiments without worrying any interfering. Once the requested time slot is up, the user is asked to log off from the system and make the system resources available to the other users.

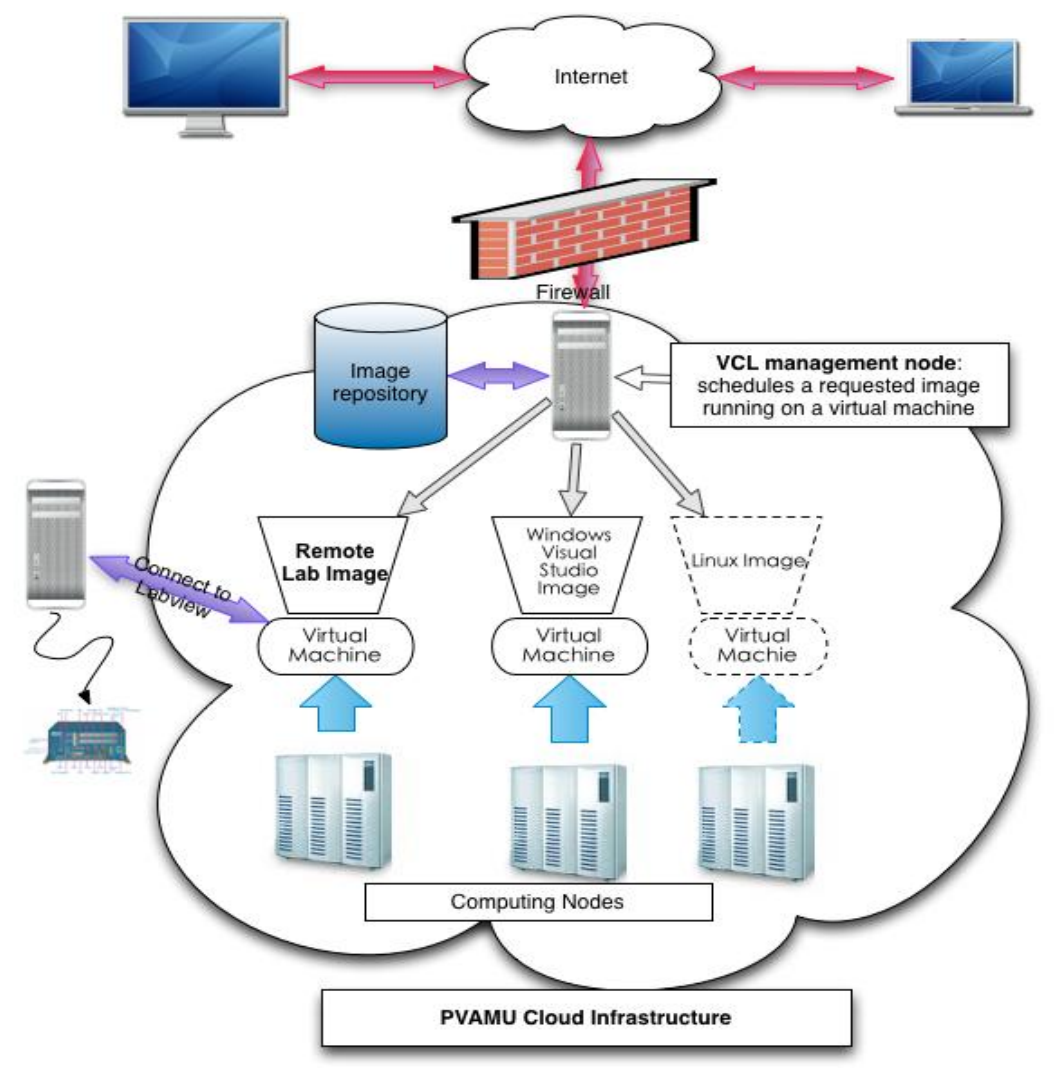

Figure 3. The new Cloud-based engineering remote laboratory

It should be noted that once the Cloud launches the user requested image to a dedicated computer environment, the user will no longer contact the VCL management node. It will directly "talk" with the assigned computer using Remote Desktop and VPN. So the managing node will serve other requests and focus on resources arrangement. This scheme effectively solved the bottleneck problem in the previous remote lab framework.

The benefits of the new remote lab design include the following:

1. Utilize the sophisticated Cloud scheduling function to enable convenient lab reservations.

2. Provide a dedicated and complete remote lab environment to enable more complex experiments using the full functionality of LabVIEW.

3. Support a diversity of lab platforms and applications through VMware virtual machine

4. Share the Cloud resources, and provide a cost-efficient solution.

5. Provide a secured and centralized management environment.

\section{Project Implementation and Cloud Enhancement}




\section{A. Project Activities}

This project is implemented following a phased plan. In the first stage, we pilot tested online remote labs by incorporating previous research outcomes-the scheduler web server scheme (Figure 1). We revamped the teaching materials for a core engineering course ELET4623 Mixed Signal (II) and its associated lab session ELET4621 Mixed Signal (II) lab. LabVIEW-based DSP hardware learning modules were developed and applied in teaching activities. Student performed three experiments on the Speedy-33 DSP hardware for impulse response, convolution integration, and frequency response. One experiment is conducted online to remotely operate the DSP board located in another building (as shown in Figure $4 \mathrm{a}$ and $4 \mathrm{~b}$ ). The feedback is very encouraging ${ }^{8}$. Many students felt excited about the lab designs. Most students felt that the remote lab could provide them with more learning opportunities and improve their course performance.

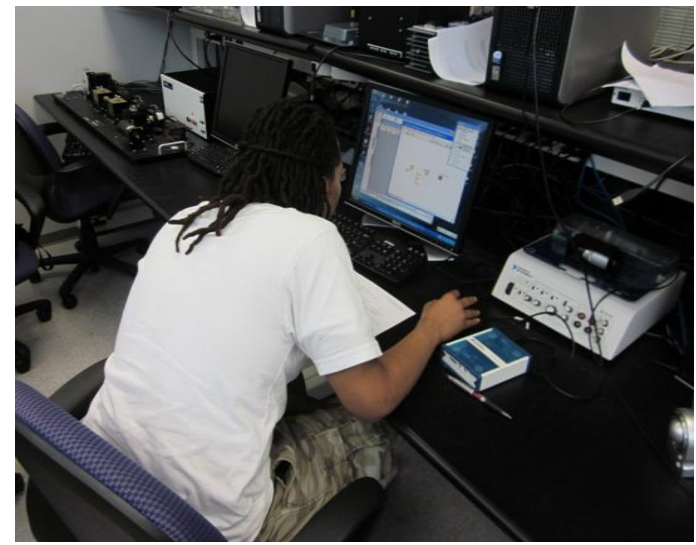

Figure 4a. Undergraduate student utilizing LabVIEW to program the NI Speedy-33 DSP board

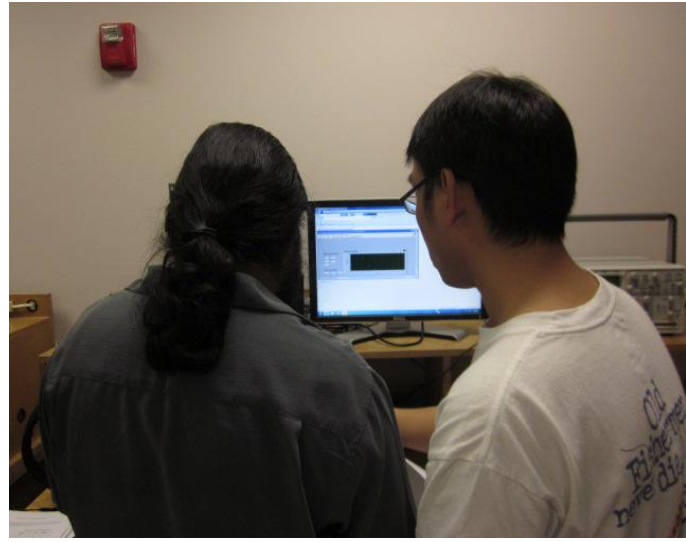

Figure $4 b$. Student programmed DSP is connected via internet to perform experiment from another room

Based on the first stage outcomes, a faculty workshop was organized in 2011 summer to engage more colleagues into using the latest teaching technologies, and to increase the exposure of the VR-Lab facilities to neighbor academic community (as shown in Figure 5a and 5b). Four faculty attendees received training, and the authors gave presentations on the online laboratory development, Cloud Computing, and online testing/training management system.

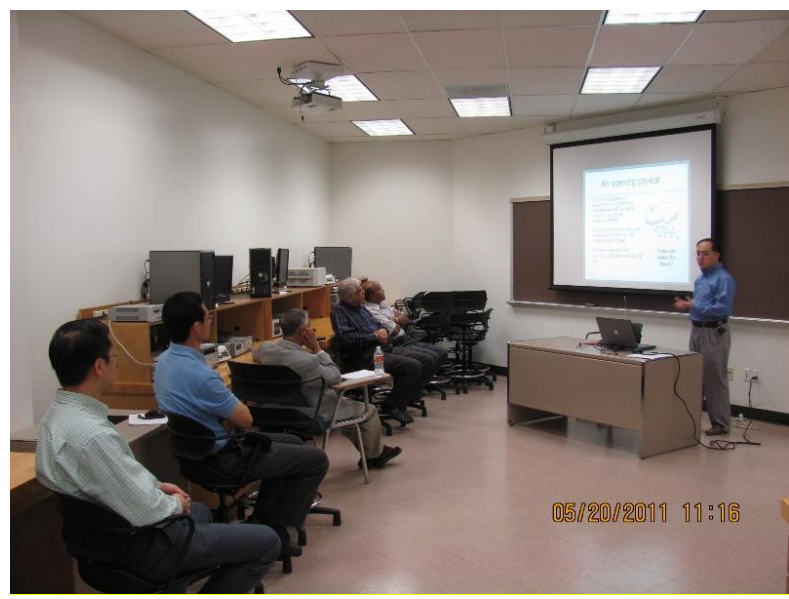

Figure 5a. Dr. Huang presenting Cloud Computing and its potential utilization in online education

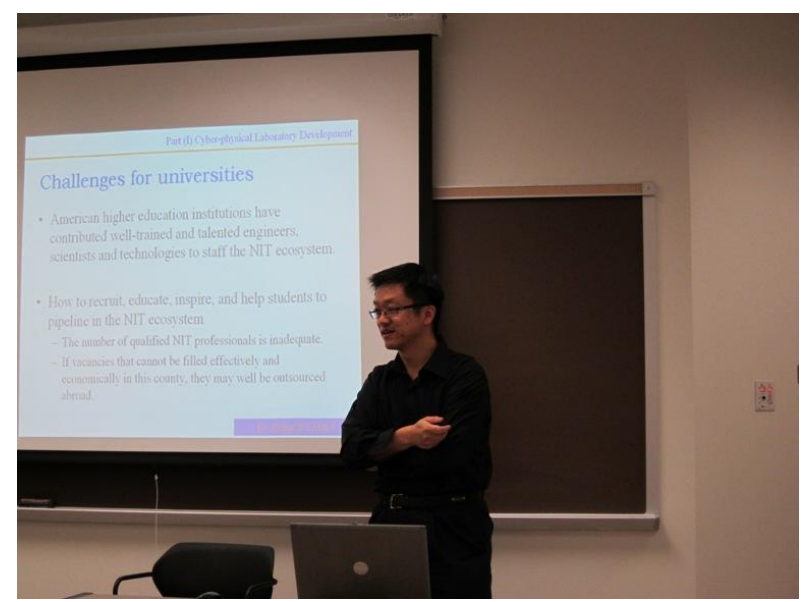

Figure 5b. Dr. Zhang presenting the development of online laboratories 


\section{B. VR-Lab with Cloud Enhancement}

In the next stage, more engineering laboratories will be made online by using SWS, and multiple laboratories will be configured so that they can be operated concurrently. Meanwhile, we are working to integrate the VCL infrastructure into the VR-Lab design to benefit more subjects and more students. An illustration of lab scheduling through VCL and one VR-Lab example is shown in Figure 6 and Figure 7.

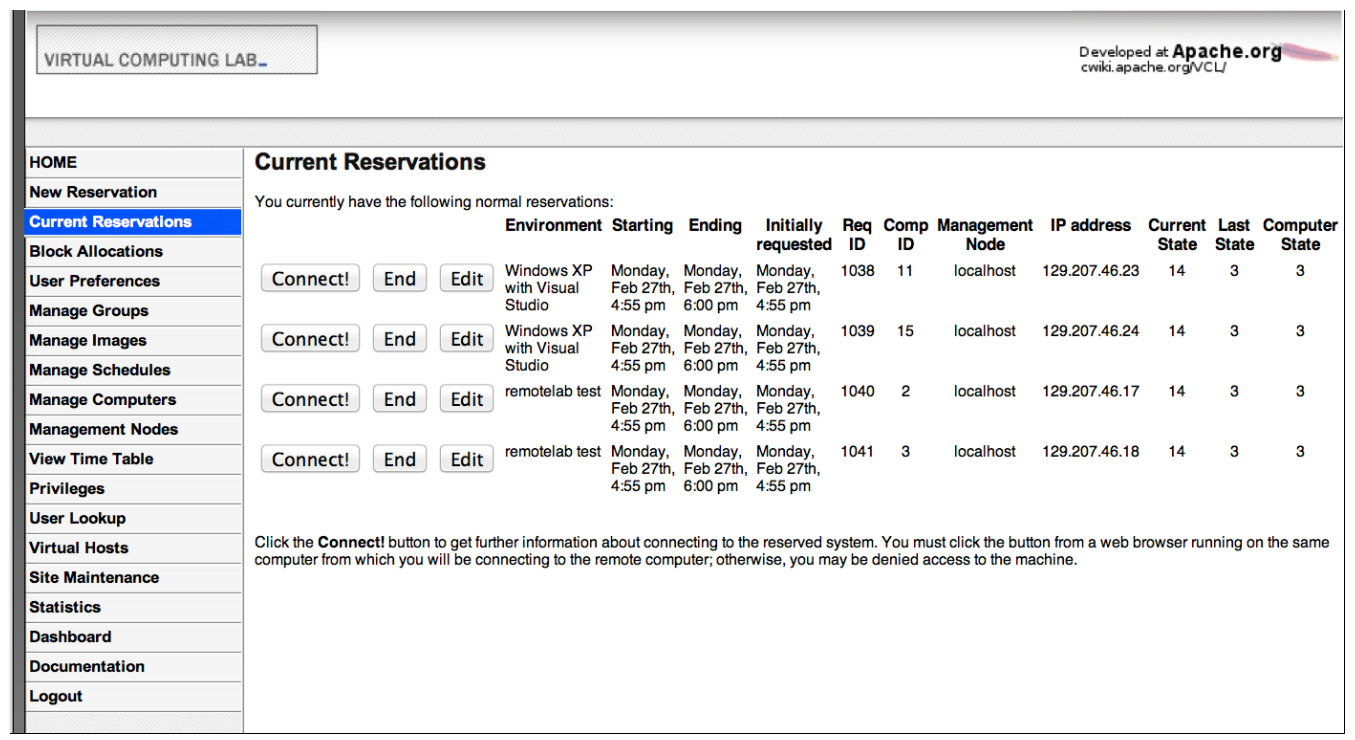

Figure 6. Lab scheduling in VCL
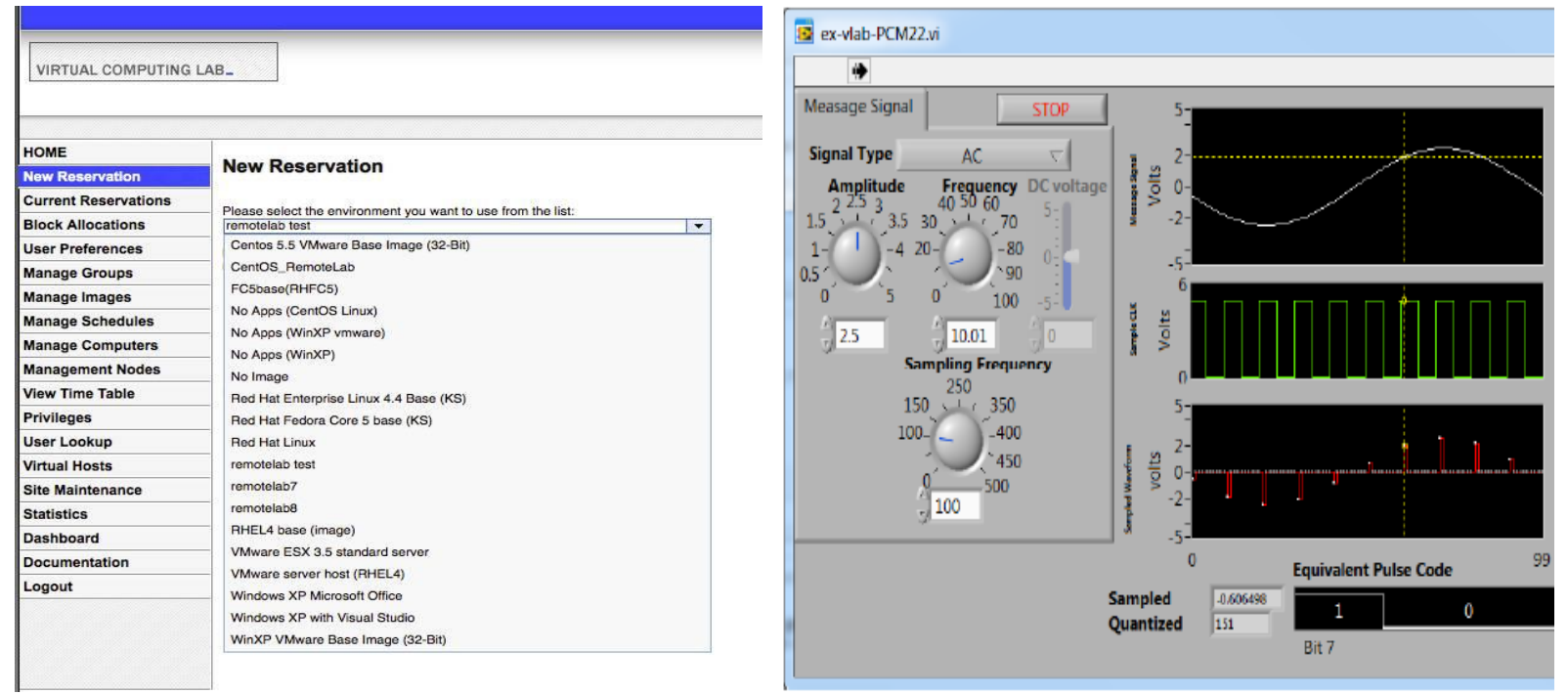

Figure 7. Virtual machine selection and remote lab operation

At this stage, we will collect and compare course assessment data before and after applying the educational innovation to show the differences in teaching effectiveness. Utilizing the lab infrastructure, we will also investigate new teaching methodologies in engineering and technology education. 


\section{Conclusions and Future Work}

This paper reviewed the current activities of a NSF sponsored project to develop remote and virtual laboratories for Engineering Technology program. The goal is to enhance engineering lab delivery efficiency and provide students with better learning opportunities. In the next project year, Cloud Computing and the developed Scheduler Web Server will be incorporated into more subjects. The researchers at PVAMU will collaborate with neighboring institutions to share their courseware in corresponding lab courses. At the same time, the related faculties will collaborate to seek further support for sustainable project development.

\section{Acknowledgment}

This project is supported in part by National Science Foundation CCLI Type I award \# 0942807. Opinions, findings, and conclusions or recommendations expressed in this material are those of the authors and do not necessarily reflect the views of the National Science Foundation.

\section{Bibliography}

1. Allen, I. E.; Seaman J., "Class Difference: Online Education in the United States, 2010”, Sloan Consortium of Individual, Institution and Organizations Committed to Quality Online Education, http://www.sloanc.org/publications/survey/staying_course, 2010

2. Bell, J. T.; Fogler, H. S., "Virtual Reality Laboratory Accidents", Proceedings of the American Society for Engineering Education (ASEE) Annual Conference and Exposition, Albuquerque, New Mexico, June 2001

3. Valera, A.; Diez, J. L.; Valles, M.; Albertos, P., "Virtual and Remote Control Laboratory Development", IEEE Control Systems Magazine, pp. 35- 39, February 2005.

4. Chen, X.; Song, G.; and Zhang, Y., "Virtual and Remote Laboratory Development: A Review," Proceedings of Earth and Space 2010, Honolulu, HI, pp. 3843-3852, March 2010

5. Creeger, M., "Cloud Computing: An Overview", ACM Queue Distributed Computing, vol. 7, no. 5, 2009

6. Vouk, M.A, "Cloud computing - Issues, research and implementations", Proceedings of 30th International Conference on Information Technology Interfaces, pp.31-40, June 2008

7. Chen, X.; Jiang, L.; Darayan S.; Kehinde, L.; and Olowokere, D., "Technologies for Development of Virtual and Remote Laboratories - A Case Study," Proceedings of ASEE Annual Conference \& Exposition, Austin, TX, June 2009

8. Zhang, Y.; Li, L.; and Chen, X., "Virtual and Remote Functionality Development for Undergraduate Laboratory", Proceedings of ASEE Annual Conference, Vancouver, Canada, June, 2011

9. Schaffer, H. E.; Averitt, S. F.; Hoit, M. I.; Peeler, A; Sills, E. D.; Vouk, M. A.; "NCSU's Virtual Computing Lab: A Cloud Computing Solution”, Computer, vo1.42, no.7, pp.94-97, July 2009

10. Vouk, M.A., et al, "Using VCL technology to implement distributed reconfigurable data centers and computational services for educational institutions", IBM Journal of Research and Development, Vol. 53, Issue 4, pp. 2.1-2.18, July 2009 